\title{
Construcción del sentido del trabajo autónomo en jóvenes profesionistas del estado de Querétaro
}

\section{Building of the sense of autonomous work in young professionals from the state of Querétaro}

\author{
Ana Karina Calixto Guevara \\ ORCID: 0000-0001-9417-2898/karina.calixto09@gmail.com \\ Maestría en Estudios Multidisciplinarios sobre el Trabajo, Unidad Multidisciplinaria de Estudios Sobre el Trabajo (UMEST), Facultad de Psicología, \\ Universidad Autónoma de Querétaro (UAQ)
}

\section{Rolando Javier Salinas García}

ORCID: 0000-0003-0307-258X/javier.salinas.uaq@gmail.com

Profesor-investigador, UMEST, Facultad de Psicología, UAQ

\section{RESUMEN}

En los últimos años se han dado cambios y transformaciones sociales, económicas y políticas que han influido fuertemente en el campo laboral, por lo que nuevas formas de trabajo han ido surgiendo como respuesta a las nuevas dinámicas que se presentan. Los cambios en la estructura socioeconómica implementadas a partir de la flexibilidad, precarización y diversificación profesional han dado paso a formas de trabajo atípico. A partir de ello las relaciones y estructuras laborales han evolucionado desplegando diversas dinámicas, una de ellas es el trabajo autónomo. La presente investigación tiene como objetivo visibilizar en torno al sentido del trabajo que se da a esta creciente forma de trabajo atípico, específicamente se concentra en los jóvenes adultos profesionistas. En este sentido se busca explorar formas diversas en que el trabajo puede ser significado y experimentado en su cotidianidad.

\section{PALABRAS CLAVE}

trabajo autónomo, jóvenes adultos, sentido del trabajo, profesionistas
ABSTRACT

In recent years there have been social, economic and political changes and transformations that have strongly influenced the labor field. As a consequence, new forms of work have emerged in response to the new dynamics that arise. The changes in the socioeconomic structure implemented from the flexibility, precarization and professional diversification have given way to atypical forms of work. Since then, labour relations and structures have evolved and various dynamics have been deployed, one of which is self-employment. This research aims to make visible the sense of working that is given to this growing form of atypical work. It focuses specifically on young professional adults. In this sense, it seeks to explore different ways in which work can be signified and experienced in its everyday life.

\section{KEY WORDS}

autonomous work, young adults, sense of working, professionals 


\section{Introducción}

En los últimos años se han dado cambios y transformaciones sociales, económicas y políticas que han influido fuertemente en el campo laboral, por lo que nuevas formas de trabajo han ido surgiendo como respuesta a estas nuevas dinámicas. Esos cambios en las estructuras socioeconómicas, implementados a partir de la flexibilidad, precarización y diversificación profesional, han dado paso a formas de trabajo atípico. A partir de ello, las relaciones y organizaciones laborales han evolucionado desplegando diversas dinámicas, una de las cuales es el denominado trabajo autónomo.

El trabajo autónomo se ha categorizado desde los estudios laborales como una variante de los trabajos atípicos, de autoempleo y trabajo por cuenta propia, el cual debe contar con la característica de ser multifuncional, además de que el producto físico será sustituido por el trabajo inmaterial en muchos casos. El trabajo ha evolucionado y con ello ha mostrado nuevas estructuras y formas de organización, como la actividad laboral autónoma, la cual se ha presentado como una modalidad entre la población adulta joven que busca insertarse en el mercado profesional.

En ese sentido, Rueda (2016) menciona que se han construido nuevas modalidades en las que algunas condiciones laborales, como la jornada de trabajo concreta, el espacio laboral definido y los salarios establecidos, se difuminan y dan paso a estructuras heterogéneas cada vez más pertinentes, como el trabajo autónomo. Por su parte, De la Garza (2012) señala que estas nuevas formas de trabajo se caracterizan por su componente simbólico y pueden predominar sobre las objetivaciones físicas de los productos. Las nuevas formas de trabajo tienen que ver con su carácter subjetivo y simbólico y, a partir de ello, se abonará una cualidad que caracteriza las relaciones laborales: la reproducción social de la fuerza de trabajo.

En México, como lo señala Banda y Mendoza (2020), el porcentaje de trabajadores autónomos duplica con $26.8 \%$ el promedio de la Organización para la Cooperación y el Desarrollo Económico (OCDE) en 2019, con 14.2\%. Ante este contexto, el mercado laboral, para un sector de la población, se encamina hacia trabajos parciales o temporales, mucho más flexibles, en el cual los trabajadores tienen libertad para gestionar sus carreras, aprovechando sus competencias y reduciendo sus exigencias de seguridad laboral (Fernández, 2018), lo que da lugar en ocasiones a trabajos cada vez más precarizados.

\footnotetext{
${ }^{1}$ De la Garza (2009) señala que la discusión acerca del trabajo atípico tiene detrás la comparación con un tipo ideal de trabajo, como el industrial, estable, subordinado a un solo patrón, con relaciones claras y una jornada de trabajo establecida. En contraposición, atípico sería el no subordinado a un solo patrón o integrado a una sola empresa, sin contrato por tiempo indeterminado, sin tiempo completo, desprotegido, riesgoso, pero no necesariamente precario, así como también aquellos en los que el cliente está implicado en la producción.
} 
Frente al escenario que se vive en este momento, la ciudad de Querétaro no se encuentra exenta de las transformaciones y efectos que se gestan en torno al contexto laboral. Por lo tanto, es pertinente voltear la mirada a la situación que enfrentan los jóvenes y, en particular, los jóvenes adultos con formación académica² que radican en esta entidad, para identificar y comprender las dinámicas que se construyen a partir de esta situación.

El objetivo de la investigación de la cual se desprende este artículo fue explorar la construcción subjetiva y relacional de los vínculos sociales que promueven el desarrollo de estructuras personales, sociales y laborales que dotan de identidad a los sujetos, a partir del contexto laboral, social, económico y educativo en que se desarrollan los jóvenes adultos, con la finalidad de distinguir las relaciones que se han gestado en su cotidianidad y que han permeado su proceso como trabajadores autónomos.

Teniendo en cuenta lo anterior, esta investigación se realizó desde un enfoque cualitativo y la importancia de plantearla desde esta metodología apuntó a una comprensión de la realidad que tomara en cuenta procesos históricos y capacidades sociales desde una mirada subjetiva, rescatando la singularidad y particularidad de los procesos mismos (Galeano, 2004).

Para tales efectos, el estudio estuvo integrado por diez jóvenes adultos profesionistas que radican en el estado de Querétaro; cinco varones y cinco mujeres. Todos se desempeñaban como trabajadores autónomos en áreas profesionales, como ciencias y humanidades (psicólogos, antropólogos), administrativas (administración y contabilidad) e ingenierías (biosistemas), y rondaban entre los 27 y 37 años de edad.

La metodología implementada en la investigación parte de una perspectiva cualitativa, por lo que la representatividad de los datos no se da en función de una muestra representativa de la población de estudio, sino del abordaje cualitativo a través de técnicas de entrevistas semiestructuradas y a profundidad. Cabe señalar que, en un primer momento, se realizó un abordaje del fenómeno a través de una validación cualitativa del instrumento de recolección de información, cuyos resultados señalaron tres niveles de aproximación: individual, laboral y social. Este recurso permitió que se desarrollaran las entrevistas de manera puntual y se construyeran las categorías de análisis.

A partir de las respuestas de los entrevistados y en relación con los tres niveles de aproximación, se construyeron categorías que posibilitaron el acercamiento a la comprensión individual que los sujetos asumen en torno al sentido del trabajo en sus vidas. Algunas de las categorías y su abordaje fueron las siguientes (tabla 1):

\footnotetext{
${ }^{2}$ La categoría de joven adulto se aborda a partir del rango de edad que comprende de los 23 a los 39 años y teniendo como característica la formación académica profesional, destreza y habilidades en el uso de tecnología, así como un estrato social medio.
} 
Tabla 1

Categorías y abordaje analítico

\begin{tabular}{|l|l|}
\hline Categorías & Abordaje \\
\hline $\begin{array}{l}\text { Crecimiento personal } \\
\text { y proyecto de vida }\end{array}$ & $\begin{array}{l}\text { El trabajo ocupa un lugar primordial en la vida de los individuos, al contribuir } \\
\text { en la formación de su identidad. A partir de la ejecución de metas y objetivos } \\
\text { laborales, estos concretan proyectos personales que aportan al crecimiento } \\
\text { individual a través del crecimiento profesional. }\end{array}$ \\
\hline Proceso productivo & $\begin{array}{l}\text { Actividad atípica y heterogénea que sigue pautas y características individuales, } \\
\text { pero que responde a tiempos, espacios y actividades establecidos personalmente. }\end{array}$ \\
\hline Contexto social & $\begin{array}{l}\text { Los individuos se construyen y definen a partir de sus experiencias, su formación y } \\
\text { su crecimiento, y los elementos que se adquieren forjan y constituyen un sentido } \\
\text { de identidad al momento de ingresar en el mercado laboral. }\end{array}$ \\
\hline Formación académica & $\begin{array}{l}\text { La educación se asume como un momento de aprendizaje en el que se aprenden } \\
\text { las bases que, posteriormente, servirán en la creación de estrategias en los } \\
\text { contextos profesionales. }\end{array}$ \\
\hline Sentido del trabajo & $\begin{array}{l}\text { Se da a partir de la interrelación y percepción que cada uno de los jóvenes adultos } \\
\text { experimenta de manera particular, sus relaciones sociales, su crianza y educación, } \\
\text { así como de sus experiencias laborales. }\end{array}$ \\
\hline
\end{tabular}

Fuente: Elaboración propia.

La pertinencia en la investigación de tales categorías es que éstas se analizan de manera relacional con las subjetividades y percepciones que se gestan en los niveles social, individual y laboral donde confluyen los sujetos, en concordancia con la experiencia cotidiana, y en consecuencia, se observa de qué forma construyen los trabajadores el significado del trabajo autónomo.

\section{El mercado de trabajo en Querétaro: principales indicadores}

El estado de Querétaro se ha posicionado en los últimos años como un polo de creación de empleos, pero esto no significa que las transformaciones y la constante evolución del mercado de trabajo no impacten en las dinámicas laborales de la entidad. En este sentido, como lo señala Banda (2019), para el primer mes de 2019 se registró una tasa de desocupación del 3.6\%, lo que representa cerca de 31 mil personas en busca de un empleo formal, conforme lo indica la Encuesta Nacional de Ocupación y Empleo (ENOE) del Instituto Nacional de Estadística y Geografía (INEGI, 2019).

Esta situación enmarca una de las problemáticas más importantes a las que se enfrenta el país. La situación laboral impacta cualquier contexto en la vida de las personas, afectando a todos los sectores poblacionales. En la presente investigación nos enfocamos en los jóvenes adultos profesionistas de la entidad. Respecto a ello, la Federación de Colegios y Asociaciones de Profesionistas del Estado de Querétaro (FECAPEQ) señala la problemática de empleo que han enfrentado en la primera mitad del año las diferentes disciplinas ahí 
agrupadas, y que, como consecuencia, ha generado el creciente subempleo ante la necesidad de ingresos económicos (Banda, 2019).

Frente a esta situación, el 48\% de los jóvenes egresados de las universidades en Querétaro buscan crear su propia compañía, pero de éstos sólo el 13\% lo logra y únicamente el 3\% subsiste a largo plazo (Almanza, 2016). Teniendo en cuenta lo anterior, se presenta como consecuencia la creación de nuevas dinámicas que impactan en las estructuras laborales, las relaciones sociales y el proceso de trabajo, y por lo tanto, la construcción de los significados que se dan a partir de la experiencia en torno al trabajo autónomo se deberá abordar a partir de la cotidianidad en la que se desenvuelven los jóvenes adultos profesionistas.

\section{Construcción del sentido e identidad del trabajo autónomo}

Los individuos se forman a lo largo de su vida a partir de experiencias, del contexto en el que se desenvuelven, la familia, la carga cultural dada a partir de las tradiciones, los usos y costumbres, la educación, entre otros. Todo ello va gestando en lo cotidiano de las personas un deber ser que es parte indisoluble de la personalidad e identidad de un sujeto. Por lo tanto, el contexto, así como las condiciones sociales, económicas, políticas y educativas, tienen un papel importante en la construcción de dicha personalidad.

En este sentido, el trabajo se articula y constituye en interacción con los proyectos de otros ámbitos de la vida, donde las distintas esferas vitales de los jóvenes adultos interactúan y otorgan significado conjuntamente a las decisiones y proyecciones laborales dadas por la subjetividad, sin olvidar que el principal motor de esto proviene de un proyecto de vida articulado con el mejoramiento de la calidad de vida (Roberti, 2016). El trabajo autónomo permite a los sujetos, desde su apreciación, retomar la centralidad del trabajo en la vida y en su cotidianidad y acceder a otras áreas de la vida donde la creatividad y la innovación se prestan para redefinir el trabajo.

Los constantes cambios en el contexto laboral han traído consigo importantes restructuraciones que han impactado en la manera en como los individuos perciben el trabajo actualmente; sin embargo, el trabajo autónomo se ha resignificado a partir de nuevos conceptos que se acercan a las emergentes modalidades atípicas del trabajo (Pereira, 2013), es decir, se ha trasformado y diversificado adoptando nuevas formas y relaciones laborales que impactan no sólo en la vida productiva de los sujetos, sino también en la personal y social.

Una de las razones por las cuales los jóvenes adultos se están insertando en esta nueva modalidad responde a un deseo de crecimiento no sólo profesional sino personal, es decir, se persigue una forma alternativa de concebir el trabajo a partir de la subjetividad, imbuido de anhelos, significados y deseos. La autorrealización tiene una función determinante al momento de asumirse como autónomos. Las implicaciones sociales y económicas que conlleva realizar este tipo de trabajo pueden poner en juego la decisión de seguir o ingresar 
en algún trabajo típico; sin embargo, cuando se alcanzan las metas y objetivos planteados, para muchos se trata de la validación de su decisión.

Es por ello que el sentido e identidad que da el trabajo autónomo a los jóvenes adultos se construye no sólo a partir del contexto, también surge del capital social, económico y académico y esto da la pauta para crear relaciones que les ayudan a construir su proceso laboral como autónomos y les permiten identificarse en estas nuevas dinámicas profesionales como sujetos activos del mercado laboral.

\section{El trabajo autónomo y la construcción social}

A raíz de la investigación se detectó que el trabajo autónomo se construye a partir de las relaciones personales y laborales, las cuales se entrelazan y complementan con base en las subjetividades que confluyen en esas relaciones. En éstos se gestan los significados sociales que construyen a los sujetos a lo largo de su vida y que hoy en día los conforman y definen. Por lo tanto, los individuos se encuentran sujetos tanto a las representaciones personales y laborales como a las sociales.

No existe una regla para determinar las razones por las que algunos jóvenes deciden formarse en una u otra carrera universitaria. Se puede señalar que las decisiones laborales asumidas después de egresar se relacionan con las posibilidades familiares, es decir, se considera que los jóvenes que adquieren altos niveles de escolaridad provienen de familias mejor situadas socioeconómicamente, que les permiten afrontar durante periodos más largos los costos asociados a la búsqueda de un trabajo que cubra sus expectativas (Márquez, 2011).

Teniendo en consideración que las relaciones sociales se entrecruzan con aspectos familiares, personales, laborales, ideológicos, entre otros, no cabe duda de que no se puede abarcar la realidad social desde un sólo panorama, pues ésta es mucho más compleja; pero para comprenderla es necesario observar desde la cotidianidad y la subjetividad las relaciones, los vínculos y las actividades que día a día los individuos realizan.

De igual manera, se debe observar que la posición social de los sujetos está dada a partir de una estructura socioeconómica particular, la cual presenta desigualdades para diversos grupos poblacionales. La realidad que enfrentan los jóvenes adultos no es la misma para todos: si bien para algunos la reproducción del trabajo autónomo ha sido posible gracias al apoyo y respaldo de sus relaciones familiares y sociales, para otros se ha tratado de un trabajo en solitario, con mayores dificultades y en ocasiones mayor incertidumbre. El contexto de los jóvenes adultos con estudios profesionales cada día es más complejo. Por ello se debe tener en cuenta la situación personal, económica y social de los jóvenes, ya que las condiciones de existencia influyen en la manera de construir el sentido laboral (Guzmán, 2004).

Observar las prácticas de la vida social que se reproducen en la cotidianidad de los sujetos permite dar cuenta del sentido que se otorga a las actividades diarias, es decir, las 
prácticas religiosas, políticas, culturales, económicas, educativas, entre otras, cuyas distintas expresiones, modos de sentir, percibir e interpretar el mundo dan sentido a la realidad subjetiva e impactan en la colectividad (Pereira, 2013).

Es importante destacar que la configuración tanto del significado del trabajo como del sentido que se le da a la profesión surge de la socialización que se hace de éste. Por lo tanto, en el caso del trabajo autónomo, en el que las relaciones interpersonales son escasas y las interacciones son virtuales y a distancia, el sentido se da a partir de las sensaciones, proyecciones y satisfacciones que otorga el desarrollo de esta actividad laboral.

Socialmente el trabajo sirve para atender necesidades materiales, así como la creación de relaciones personales; también es un elemento de cohesión de las personas en la sociedad y, en el caso de este sector laboral, se da a partir del sentido de libertad que ofrece, es decir, de la gestión del tiempo que permite la socialización, la recreación, la reproducción de relaciones, entre otras. Si bien las relaciones laborales podrían ser escasas, este tipo de trabajo da acceso a tiempos y espacios para la recreación y el ocio y, por lo tanto, para la vida personal y social, las cuales tienen un papel importante al momento de ejercer dicha profesión, incluso como una de las razones para continuar con la autonomía laboral.

\section{Reflexiones finales}

Con respecto a lo mencionado, el sentido del trabajo autónomo entre la población joven adulta se construye a partir de las relaciones que se gestan en la vida cotidiana, así como de las percepciones que se dan a raíz de su identidad como autónomos, y cómo esto impacta en su contexto y vida privada. Todo ello en el entendido de que, si bien se trata de una forma relativamente nueva en el contexto laboral, ésta se encuentra también sujeta a relaciones sociales, económicas, políticas, culturales, entre otras, y por lo tanto, la actividad laboral se configura en torno a ellas.

Se puede observar como diversos factores impactan en la subjetividad que se tiene en el desempeño de las propias actividades, a partir del análisis de los significados que se construyen, así como de los elementos en los cuales se apoyan para resignificar el trabajo a corto, mediano y largo plazo. Los distintos elementos como la incertidumbre, la autorrealización, el desencanto, el sentido de libertad, entre otros, pueden desempeñar un papel importante en la creación de su identidad.

Todo ello aporta herramientas y recursos que visualizan el sentido del trabajo como un acto tanto individual como colectivo, en virtud de la búsqueda del crecimiento profesional, personal, familiar e incluso social. Se puede establecer que la construcción del sentido del trabajo autónomo en jóvenes adultos se va dando en torno a una serie de relaciones, significados y articulaciones subjetivas, entre muchos otros valores y categorías que se entrelazan para dar paso a las significaciones que se desarrollan día a día en su contexto cotidiano. 
El trabajo, por lo tanto, es visto como un componente vital y rector del crecimiento personal y profesional de los jóvenes, y se construye a partir de un proyecto de vida y de la visión de futuro que tienen de ellos mismos; sin embargo, no se debe olvidar que esta autopercepción se complementa por las relaciones familiares, la carga cultural, el entorno, la educación, las experiencias, entre otros.

\section{Referencias}

Almanza, L. (6 de julio de 2016). Apoyarán a jóvenes emprendedores de Querétaro. El Financiero. https://www.elfinanciero.com.mx/bajio/apoyaran-a-jovenes-emprendedores-dequeretaro.html

Banda, L. (29 de febrero de 2019). Registra el estado tasa de desocupación del 3.6\%. Diario de Querétaro. https://www.diariodequeretaro.com.mx/local/registra-el-estado-tasa-dedesocupacion-del-3.6-por-ciento-3122473.html

Banda, D. y Mendoza, A. (2020). Excelencia educativa. Ventana. Escuela Bancaria y Comercial. (102), 17-18. http://www.materialesebc.mx/ventana/102/ventana ebc num102.pdf

De la Garza, E. (2009). Hacia un concepto ampliado del Trabajo. En J. C. Neffa, E. De la Garza y L. Muñiz (Eds.), Trabajo, empleo, calificaciones profesionales, relaciones de trabajo e identidades laborales (pp. 111-114). Consejo Latinoamericano de Ciencias Sociales (CLACSO). http://biblioteca.clacso.edu.ar/ar/libros/coedicion/neffa1/07.pdf

De la Garza, E. (2012). El trabajo no clásico y la ampliación de los conceptos de la Sociología del Trabajo. Revista de Trabajo. Dinámica del trabajo en el marco de la incertidumbre global, 8 (10), 109-124.

Fernández, A. (2018). La creciente tendencia hacia el trabajo autónomo. Eleconomista. https:// www.eleconomista.es/opinion-blogs/noticias/9277528/07/18/La-creciente-tendenciahacia-el-trabajo-autonomo.html

Galeano, E. (2004). Diseño de proyectos en la Investigación Cualitativa. Universidad. EAFIT.

Guzmán, C. (2004). Los estudiantes frente a su trabajo. Un análisis en torno a la construcción del sentido del trabajo. RMIE, 9 (22). 747-767.

Instituto Nacional de Estadística y Geografía (2019). Encuesta Nacional de Ocupación y Empleo (ENOE). https://www.inegi.org.mx/app/glosario/default. html?p=EHENOE15mas\#letraGloS

Márquez, A. (2011). La relación entre educación superior y mercado de trabajo en México. Una breve contextualización. Perfiles Educativos, XXXIII, (núm. especial). http://www.scielo. org.mx/scielo.php?pid=S0185-26982011000500015\&script=sci arttext

Pereira, L. (2013). La representación social del trabajo en los jóvenes universitarios. Revista Latinoamericana de Derecho Social, 17, 145-177. https://www.redalyc.org/articulo. oa?id $=429640272006$

Año 16, núm. 40, noviembre 2020-marzo 2021 
Roberti, E. (2016). Los sentidos (des)centrados del trabajo: Hacia una reconstrucción de los itinerarios típicos delineados por jóvenes. Última Década, 24 (44). http://dx.doi. org/10.4067/S0718-22362016000100009

Rueda, E. (2016). El trabajo autónomo: un análisis internacional y comparado de México, España e Italia [Tesis de doctorado, Universidad de Bérgamo].

Año 16, núm. 40, noviembre 2020-marzo 2021

ISSN: 2007-1760 (impreso) 2448-9026 (digital) | DOI: 10.30973/inventio/2021.16.40/8 